\title{
Avaliação dos Indicadores da Assistência Pré-Natal em Unidade de Saúde da Família, em um Município da Amazônia Legal
}

\section{Evaluation of the indicators of prenatal care in a family health unit in a municipality in the Legal Amazon}

Marcela de Souza Sotto Mayor ${ }^{1}$; Sávia Denise S. C. Herrera²; Michelly Queren Araujo3; Fernanda Martins dos Santos³; Rhenan Vilela Arantes ${ }^{3}$; Neilton Araujo de Oliveira ${ }^{4}$

\section{RESUMO}

Introdução: a utilização de indicadores como método de avaliação de desempenho das estratégias de redes tem sido cada vez mais utilizada no Brasil, especialmente na saúde, para gestão de políticas públicas. Objetivo: avaliar os indicadores de atenção preconizados pelo Programa de Humanização no Pré-Natal e Nascimento (PHPN), a Rede Cegonha e a adequação destes nos atendimentos de pré-natal realizados na Unidade de Saúde da Família em Gurupi-TO, município da Amazônia Legal. Materiais e Métodos: estudo epidemiológico descritivo realizado pela atenção primária da saúde de Gurupi-TO, entre março-2016 e novembro-2017, avaliadas através dos indicadores de assistência pré-natal, PHPN e Índice MS. Foram calculados frequência, média, incidência e porcentagem de 50 pacientes, por meio de consulta, prontuários e cartões de gestantes. Resultados: das gestantes, $46 \%$ completaram o objetivo preconizado do PHPN. Mas ao avaliar o componente básico da qualidade pré-natal proposto pelo Ministério da Saúde, apenas $18 \%$ de todas as gestantes apresentaram 'classificação adequada superior' e 24\% tiveram 'assistência adequada', outros 24\%, 'intermediária' e 34\% 'inadequada'. Conclusão: Além de aprimoramento das estratégias de captação precoce das gestantes, com conscientização e mobilização da população sobre a importância do acompanhamento do pré-natal, faz-se necessário registro mais acurado dos dados desses serviços.

Palavras-Chave: Avaliação em saúde. Saúde materno-infantil. Cuidado pré-natal. Programas Governamentais.

\section{ABSTRACT}

Introduction: the use of indicators as a method of performance evaluation of network strategies has been increasingly used in Brazil, especially in health, public policy management. Objective: to evaluate the warning indicators recommended by the Program of Humanization in prenatal and birth (PHPN), Stork and the appropriateness of these in prenatal care in family health unit in Gurupi-TO, municipality of Amazon Cool. Materials and methods: descriptive epidemiological study carried out by primary health attention of Gurupi-TO, between March and November 2016-2017, evaluated through the prenatal assistance indicators, PHPN and Ms. Index were calculated frequency, average, incidence and percent of 50 patients, through consultation, charts and cards of pregnant women. Results: of pregnant women, $46 \%$ completed the objective advocated the PHPN. But when assessing the basic component of quality prenatal care proposed by the Ministry of health, only $18 \%$ of all pregnant women have submitted adequate rating higher than ' and ' $24 \%$ had ' proper' assistance, other $24 \%, 34 \%$ intermediate ' and 'inadequate '. Conclusion: Besides improving early capture strategies of pregnant women, with awareness and mobilization of the population about the importance of prenatal monitoring, it is necessary to more accurate record of the data from these services.

Keywords: Health Evaluation. Maternal and child health. Prenatal care. Government programs.
${ }^{1}$ Médica especialista em Saúde da Família e Comunidade pelo Centro Universitário UnirG, Gurupi-TO, Brasil.

E-mail:

marcelassmayor@gmail.com

${ }^{2}$ Fisioterapeuta. Mestranda em Ciências da Saúde-UFT, professora assistente do Centro Universitário UnirG, Gurupi-TO.

3 Acadêmicos de medicina do Centro Universitário UnirG.

${ }^{4}$ Médico. Doutor em Ensino de Biociências e Saúde pelo Instituto Oswaldo Cruz (IOC/FIOCRUZ). Mestre em Saúde Coletiva pelo Instituto de Saúde Coletiva da UFBA. Professor da Universidade Federal do Tocantins. 


\section{INTRODUÇAOO}

A estratégia de redes na perspectiva da gestão e das políticas públicas tem sido cada vez mais frequente no Brasil, envolvendo diferentes atores e organizações vinculadas entre si a partir do estabelecimento e manutenção de objetivos comuns e de uma dinâmica gerencial compatível e adequada (FLEURY, 2008). Com o crescimento da implementação de redes de atenção à saúde, é indispensável à gestão que haja um método de avaliação do desempenho das mesmas. Isso possibilita a verificação da coerência entre mudanças no modelo de atenção e as necessidades de saúde da população. Desta forma, como uma ferramenta que possibilita esta avaliação foi proposto a aplicação de indicadores de desempenho (ARAUJO et al, 2016).

Os indicadores são medidas-síntese que possuem informação relevante sobre determinadas características e dimensões do estado de saúde e também do desempenho do sistema de saúde. Eles foram desenvolvidos para facilitar a quantificação e a análise das informações produzidas e auxiliar a avaliação da situação sanitária da população, a tomada de decisões baseadas em evidências e a programação de ações de saúde (RIPSA, 2017).

Como forma de melhorar os indicadores, ainda em 2000, o governo federal instituiu no país o Programa de Humanização no Pré-Natal e Nascimento (PHPN), com o propósito de reduzir as altas taxas de morbimortalidade materna e perinatal, adotando medidas para melhoria do acesso, da cobertura e da qualidade do acompanhamento pré-natal, da assistência ao parto e puerpério (SERRUYA, 2004).

A avaliação de programas e políticas de saúde vem ganhando espaço no Brasil e, em destaque, as políticas de saúde materno-infantil, onde a qualidade da atenção pré-natal impacta diretamente nos indicadores de saúde, contribuindo para a redução das taxas de morbimortalidade materna e perinatal. Dessa forma, é relevante a avaliação do pré-natal, principalmente em países com amplas desigualdades regionais econômicas e sociais e de acesso aos serviços de saúde (POGLIANE, 2014), como é o caso de municípios na Amazônia Legal.

Nesses casos, a atenção pré-natal pode controlar os fatores de risco que trazem complicações à gestação, além de permitir a detecção e o tratamento oportuno de complicações, contribuindo para que os desfechos perinatais e maternos sejam favoráveis (DOMINGUES et al, 2012). 
O Programa Rede Cegonha é uma estratégia do Ministério da Saúde (MS) que visa implementar uma rede de cuidados para assegurar às mulheres o direito ao planejamento reprodutivo e a atenção humanizada à gravidez, ao parto, aborto e ao puerpério, bem como às crianças o direito ao nascimento seguro e ao crescimento e desenvolvimento saudáveis. É uma estratégia de enfrentamento da mortalidade materna, da violência obstétrica e da baixa qualidade da rede de atenção ao parto e nascimento, desenvolvendo ações para ampliação e qualificação do acesso ao planejamento reprodutivo, pré-natal, parto e puerpério (BRASIL, 2011).

Desde 2011, o governo federal vem implementando a Rede Cegonha como forma de complementar o PHPN e, comisso, fomentar a implementação de um novo modelo de atenção à saúde da mulher e da criança, desde o pré-natal até a puericultura das crianças até 24 meses; organizar a rede de atenção à saúde materna e infantil, para que garanta acesso, acolhimento e resolutividade; e reduzir a mortalidade materna e infantil com ênfase no componente neonatal (BRASIL, 2011).

A avaliação dos parâmetros que compõem o PHPN e a Rede Cegonha permite inferir diretamente a qualidade de assistência pré-natal nos serviços do Sistema Único de Saúde (SUS). Sendo assim, as baixas taxas de adequação do processo pré-natal nos vários níveis podem ter, como resultado, desfechos indesejáveis como nascimento de crianças prematuras e de baixo peso, além de contribuir para mortalidade materna e perinatal (MARTINELLI, 2014).

O presente trabalho tem como objetivo avaliar os indicadores de atenção preconizados pelo PHPN e pela Rede Cegonha e a adequação destes nos atendimentos de pré-natal realizados na Unidade de Saúde da Família (USF) João Manoel dos Santos, em Gurupi- TO, município da Amazônia Legal.

\section{MATERIAIS E METODOS}

Estudo epidemiológico descritivo realizado na Unidade de Saúde da Família (USF) João Manoel dos Santos, no município de Gurupi, no sul do estado do Tocantins. Foram avaliados prontuários e cartões de gestantes que realizaram o Pré-natal, com a equipe 007, no período de março de 2016 a novembro de 2017. Esta equipe foi escolhida por ser pioneira na implantação da RC no município de Gurupi- TO e por ter realizado o acompanhamento de um ciclo completo de gestantes com atividades educativas.

As gestantes foram recrutadas, sob livre demanda, tanto durante as consultas de prénatal quanto por meio de busca ativa pelos agentes comunitários de saúde (ACS), 
realizando a assinatura do Termo de consentimento Livre e Esclarecido (TCLE) após serem informadas a respeito da pesquisa. Esta pesquisa foi autorizada pela Secretaria Municipal de Saúde do município de Gurupi- TO e se iniciou após aprovação do Comitê de Ética e Pesquisa em seres humanos, do Centro Universitário UnirG, sob o parecer 2.292.846.

A amostra foi constituída por 50 gestantes, sem restrição de faixa etária materna que tiveram a oportunidade de realizarem o pré-natal no período de março de 2016 a novembro de 2017. Foram analisados os sete indicadores de processo do PHPN para avaliação da assistência pré-natal, segundo a portaria do Ministério da Saúde (№ 569, 1ํ de Junho de 2000), sendo eles: 1-Percentual de gestantes que se inscreveram no programa e realizaram a $1^{1}$ consulta até o quarto mês; 2-Percentual de gestantes inscritas que realizaram 06 (seis) consultas de pré-natal; 3-Percentual de gestantes inscritas que realizaram 06 (seis) consultas de pré-natal e a consulta de puerpério; 4- Percentual de gestantes inscritas que realizaram 06 (seis) consultas de pré-natal e todos os exames básicos; 5-Percentual de gestantes inscritas que realizaram 06 (seis) consultas de pré-natal, a consulta de puerpério e todos os exames básicos; 6-Percentual de gestantes inscritas que receberam a dose imunizante da vacina antitetânica (DTPa); 7-Percentual de gestantes inscritas que realizaram (06) seis consultas de pré-natal, a consulta de puerpério, todos os exames básicos, o teste anti-HIV, a dose imunizante da vacina antitetânica.

Em relação ao primeiro indicador, foi considerado a realização da primeira consulta em até 12 semanas, segundo atualização das diretrizes do MS em 2012.

Após realizar a frequência dos indicadores do PHPN, foi aplicado o Índice MS adaptado por Almeida e Barros (2005) para a análise da adequação da assistência prénatal. Esse índice possui seis parâmetros: 1) Início do atendimento pré-natal: antes de 12 semanas de gestação; 2) Número de consultas de pré-natal: realização de seis ou mais consultas para uma gestação de 37 semanas ou mais; cinco consultas para a gestação com 32 a 36 semanas; e quatro consultas para gestação com 22 a 32 semanas; 3) Realização de todos os exames de rotina propostos na primeira consulta de pré-natal (Hemograma, Tipagem Sanguínea e Fator Rh, Glicemia de Jejum, Urina, Teste para sífilis e Anti-HIV, Sorologia para hepatite B e toxoplasmose); 4) Realização de pelo menos cinco procedimentos clínico-obstétricos em todas as consultas (verificação de peso, pressão arterial, batimentos cardiofetais, altura uterina e edema); 5) Orientação sobre amamentação e 6) Realização de pelo menos um exame de ultrassonografia.

O índice MS classifica a assistência pré-natal em adequada superior quando todas as seis recomendações são satisfeitas; adequada quando são cumpridas cinco 
recomendações; intermediária na presença de quatro recomendações e inadequada quando três ou menos recomendações foram satisfeitas.

Os dados foram cadastrados e tabulados através do programa Microsoft Excel 2016®. Fez-se frequência, média, incidência e porcentagem das variáveis sobre as gestantes avaliadas para as variáveis do estudo.

\section{RESULTADOS E DISCUSSAO}

Participaram da pesquisa 50 gestantes, entre 16 e 42 anos, acompanhadas na atenção primária da saúde, no período de março de 2016 a novembro de 2017 no município de Gurupi- TO. A Tabela 1 representa os indicadores do PHPN e suas porcentagens de gestantes.

Tabela 1. Número/porcentagem de gestantes de 2016 e 2017 consultadas pela USF conforme os indicadores do Programa de humanização do pré-natal e nascimento (PHPN)

\begin{tabular}{llc}
\hline Gestantes & N & $\%$ \\
\hline Iniciaram até 12 semanas & 23 & $46 \%$ \\
6 ou mais consultas & 30 & $60 \%$ \\
Vacina antitetânica DTPa & 34 & $68 \%$ \\
6 ou mais consultas e puerpério & 24 & $48 \%$ \\
6 ou mais consultas e todos exames & 29 & $58 \%$ \\
6 ou mais consultas/ puerpério/ todos exames & 24 & $48 \%$ \\
6 ou mais consultas/ puerpério/ todos exames/ vacina antitetânica DTPa & 23 & $46 \%$
\end{tabular}

Foi verificado que 29 (58\%) gestantes realizaram todos os exames laboratoriais preconizados. Estes resultados foram menores aos verificados por Polgliane et al (2014) no município de Vitória (ES) onde foi observado que 70 a $80 \%$ das gestantes realizaram os exames. A frequência de realização dos exames por parte das gestantes pode ser explicada pela facilidade de acesso à coleta, entretanto, as falhas na realização de exames pelas gestantes, se estendem desde a negligência por parte das gestantes até a demora para entrega dos resultados pelo laboratório. A realização dos exames laboratoriais durante a gestação é de suma importância para a prevenção, identificação e correção de alterações que possam prejudicar a gestante e seu concepto, bem como dar início ao tratamento de comorbidades já existentes ou que possam surgir durante a gestação (COSTA et al, 2013).

Ainda no estudo de Polgliane et al (2014), a porcentagem de gestantes que realizaram 6 ou mais consultas de pré-natal foi de 75\%, número próximo ao encontrado no estudo 
(60\%). Segundo Domingues (2012), não há ainda um consenso a respeito do número ideal de consultas de pré-natal a ser feito pelas gestantes. De acordo com o calendário de consultas preconizado pelo PHPN, recomenda-se a realização de, no mínimo, seis consultas para uma gestante de baixo risco, três delas devendo ser realizadas no último trimestre. Porém, em se tratando da $\mathrm{RC}$, o indicador de qualidade é a realização de sete ou mais consultas de pré-natal. Considerando que neste estudo a quantidade total de gestantes que realizaram 6 ou mais consultas, segundo as diretrizes do PHPN, foi de 30 (60\%) no período analisado.

Foi constatado pela OMS através de ensaios clínicos randomizados, que um pré-natal composto por um número mínimo de quatro consultas associadas à realização de intervenções específicas, baseadas em evidências científicas, possui resultado equivalente a um pré-natal com mais consultas (CARROLI et al, 2001). Contudo, um estudo publicado por Hofmeyr e Hodnett (2013) demonstrou que um cuidado pré-natal com menor quantidade de consultas apresenta maior mortalidade perinatal.

Verificou-se que, referindo-se ao início do acompanhamento pré-natal com IG até a $12^{\text {a }}$ semana, deram início neste período $23(46 \%)$ sendo este grupo inferior a $50 \%$ com resultado similar ao estudo de Polgliane et al (2014), onde cerca de $38 \%$ das gestantes iniciaram o pré-natal até a $12^{\underline{a}}$ semana.

O início precoce do acompanhamento pré-natal é de suma importância, pois possibilita o acesso a diagnósticos e terapias de muitas patologias com repercussões graves na saúde da mulher e do bebê, como anemia, hipertensão arterial, diabetes mellitus, e infecções por doenças infectocontagiosas como sífilis e HIV. Também proporciona melhor estimativa da idade gestacional e melhora do acompanhamento do desenvolvimento fetal. (DOMINGES et al., 2015). O início tardio da assistência pré-natal indica fragilidade no serviço de saúde e lacunas na captação precoce de gestantes e conscientização da população (POLGLIANE et al., 2014).

Em relação à efetivação da imunização, foi observado frequência de 23 (46\%) em referência ao total de gestantes inclusas no presente trabalho. Esses índices foram inferiores aos encontrados por Polgliane et al., (2014), cuja cobertura vacinal foi de 70,6\%, porém foram próximos aos resultados encontrados por Martinelli et al (2014) em estudo feito no município de São Mateus (ES), onde a imunização antitetânica foi administrada a $58,7 \%$ das gestantes avaliadas. A baixa cobertura da vacina infere maiores riscos ao surgimento de tétano neonatal e pode ser explicada pela inexistência de registros da dose no cartão da gestante ou no prontuário (FILHO, 2015; POLGLIANE et al., 2014). 
Acredita-se que com a implantação das atividades educativas da $R C$, as gestantes tenham sido mais sensibilizadas quanto à importância do seguimento do prénatal, sendo necessário salientar que assistência pré-natal somente conclui após a consulta puerperal, realizada apenas em $46 \%$ das gestantes no presente caso. Sendo parte da necessidade de seguimento clínico após a gestação no intuito de garantir um intervalo ideal entre gestações, protegendo a mulher e melhorando os resultados perinatais, através de orientações para planejamento familiar e maior possibilidade de identificar alterações como anemia e depressão pós-parto (DOMINGUES et al., 2012).

Dentre as atividades para a realização de um adequado acompanhamento pré-natal e assistência à gestante e à puérpera preconizadas pelo PHPN, as atividades educativas são estratégias que permitem à gestante a obtenção de informações que melhoram sua qualidade de vida e de seu concepto. Esse modelo de assistência pré-natal inclui além do atendimento individual realizado durante as consultas, oferecendo benefícios ao impactar a saúde e acrescenta-se um sentido mais amplo, assegurar o vínculo com a equipe de saúde (HOFMEYR e HODNETT, 2013).

A realização de ações educativas, no decorrer do ciclo gravídico-puerperal, é importante porque a mulher será orientada sobre os cuidados com a saúde na gestação, sobre o momento do parto, de forma positiva, sobre a amamentação, com menos riscos de complicações no puerpério e mais sucesso no cuidado com o bebê, principalmente para primigestas, que estão vivenciando uma novidade em sua vida (PEIXOTO, 2017).

A seguir, a Tabela 2 apresenta as frequências dos seis parâmetros do Índice MS por Almeida e Barros (2005) para a análise da adequação da assistência pré-natal.

Ao avaliar o componente básico da qualidade pré-natal proposto pelo Ministério da Saúde das gestantes, constata-se no presente estudo que $82 \%$ das gestantes realizaram todos exames preconizados e a maioria delas foram orientadas sobre a importância da amamentação. Observa-se também que apenas nove (18\%) gestantes avaliadas tiveram sua assistência classificada como 'adequada superior', sendo que o maior número delas 17 (34\%) pertencendo ao perfil 'inadequado'. O alto índice de inadequação encontrado devese ao grande número de abandono do seguimento da assistência. De forma divergente, em estudo de Pereira et al (2013), nenhuma gestante teve sua assistência enquadrada como 'adequada superior', bem como o índice de assistência 'adequada' se mostrou maior que nesse estudo (24\%), sendo de $34,8 \%$. 
Tabela 2. Número/porcentagem ou média de gestantes de 2016 e 2017 consultadas pela USF segundo critérios do Ministério da Saúde adaptados por Almeida e Barros (2005) ${ }^{1}$.

\begin{tabular}{lcc}
\hline Gestantes & $\mathbf{N}$ & $\% / \mathbf{M}^{\star}$ \\
\hline Início do atendimento pré-natal até $12^{\circ}$ semana & 24 & $48 \%$ \\
Número de consultas até $37^{\circ}$ semana ou mais & 323 & 6,46 \\
Número de consultas até $32^{\circ}$ e $36^{\circ}$ semana & 247 & 4,94 \\
Número de consultas até $22^{\circ}$ e $32^{\circ}$ semana & 191 & 3,83 \\
Realização de todos exames de rotina preconizados pelo MS & 41 & $82 \%$ \\
Pelo menos 5 (cinco) procedimentos clínicos e obstétricos & 30 & $60 \%$ \\
Orientação sobre amamentação & 31 & $62 \%$ \\
Pelo menos um exame de ultrassonografia & 48 & $96 \%$ \\
Adequação MS: & 50 & $\mathbf{1 0 0 \%}$ \\
Adequado superior & 9 & $18 \%$ \\
Adequado & 12 & $24 \%$ \\
Intermediário & 12 & $24 \%$ \\
Inadequado & 17 & $34 \%$
\end{tabular}

Legenda: \%/M*: porcentagem ou média do total de gestantes.

A partir de dados secundários coletados pelo Ministério da Saúde em 2013, Leal et al (2015) avaliou a adequação da assistência pré-natal em municípios do Nordeste e da Amazônia Legal. Foram analisadas a adequação de 5079 assistências pré-natais nos oito estados constituintes da Amazônia Legal, segundo os critérios de PHPN e descritas 2,9\% como 'adequadas' e 6,8\% como 'inadequadas' representaram 6,8\% resultados comparativamente inferiores aos encontrados neste estudo. Ainda, em se tratando do Estado do Tocantins, relatou-se que 5,6\% das assistências pré-natais foram 'adequadas', e $4,1 \%$ 'inadequadas', mantendo valores inferiores aos encontrados neste estudo.

\section{CONCLUSÁO}

Pode-se concluir neste estudo que o uso de métodos avaliativos é indispensável para o aprimoramento das estratégias de captação precoce das gestantes através da busca ativa pelos Agentes Comunitários de Saúde (ACS) e da conscientização da população sobre a importância do início precoce do acompanhamento pré-natal, assim como a importância de manter registro detalhado dos dados nos prontuários e cartões das gestantes. Além disso, sabendo-se que o pré-natal não se baseia em apenas consultas e solicitação de exames, realizar estratégias que envolvam o acolhimento e o reconhecimento das necessidades de saúde, contribui com o estabelecimento de vínculos da gestante com a equipe de saúde. 


\section{REFERÉNCIAS}

ALMEIDA, S. D. M., Barros M. B. A. Eqüidade e atenção à saúde da gestante em Campinas (SP), Brasil. Revista Panamericana de Salud Publica, v.17, n.1, p.15-25,2005.

ARAUJO, Daiane Ellwanger et al. Avaliação de desempenho das redes de atenção à saúde: uma proposta de indicadores. 2016.

BRASIL. Ministério da saúde. Secretaria de atenção à saúde. Departamento de ações programáticas estratégicas. Portaria no 1459 de 24 de junho de 2011. Institui no âmbito do SUS a Rede Cegonha. Brasília, 2011.

CARROLI, Guillermo et al. WHO systematic review of randomized controlled trials of routine antenatal care. The Lancet, v. 357, n. 9268, p. 1565-1570, 2001.

COSTA, Christina Souto Cavalcante et al. Características do atendimento pré-natal na Rede Básica de Saúde. Revista Eletrônica de Enfermagem, v. 15, n. 2, p. 516-22, 2013.

DE SOUZA, Viviane Barbosa; ROECKER, Simone; MARCON, Sonia Silva. Ações educativas durante a assistência pré-natal: percepção de gestantes atendidas na rede básica de Maringá-PR. Revista Eletrônica de Enfermagem, v. 13, n. 2, p. 199-210, 2011.

DO CARMO LEAL, Maria et al. Atenção ao pré-natal e parto em mulheres usuárias do sistema público de saúde residentes na Amazônia Legal e no Nordeste, Brasil 2010. Revista Brasileira de Saúde Materno Infantil, v. 15, n. 1, 2015.

DOMINGUES, Rosa Maria Soares Madeira et al. Adequação da assistência pré-natal segundo as características maternas no Brasil. Revista Panamericana de Salud Pública, v. 37 , p. 140-147, 2015.

DOMINGUES, Rosa Maria Soares Madeira et al. Avaliação da adequação da assistência pré-natal na rede SUS do Município do Rio de Janeiro, Brasil. Cadernos de Saúde Pública, v. 28, p. 425-437, 2012.

FILHO, D.A.S. Qualidade da assistência pré-natal na estratégia saúde da família em Duque de Caxias-RJ. Duque de Caxias. Monografia [Especialização em Saúde da Família] Universidade Estadual do Rio de Janeiro, 2011.

HOFMEYR, G. Justus; HODNETT, Ellen D. Antenatal care packages with reduced visits and perinatal mortality: a secondary analysis of the WHO antenatal care trial-Comentary: routine antenatal visits for healthy pregnant women do make a difference. Reproductive health, v. 10, n. 1, p. 20, 2013.

MARQUES QUEIROZ, Dayanna Joyce; BEZERRA SOARES, Danielle; ARAÚJO NASCIMENTO DE OLIVEIRA, Kátia Cristina. Avaliação da assistência pré-natal: relevância dos exames laboratoriais. Revista Brasileira em Promoção da Saúde, v. 28, n. 4, 2016.

MARTINELLI, Katrini Guidolini. Adequação do processo da assistência pré-natal segundo os critérios do Programa de Humanização do Pré-natal e Nascimento e Rede Cegonha. CEP, v. 29040, p. 090, 2014.

PEIXOTO, Tayná Barros. Adesão da vacinação pela gestante no pré-natal: revisão integrativa. 2017. 
PEREIRA, Nayanna Moura; GUIMARÃES, Bruna Natali Soares; LANZA, Fernanda Moura. Avaliação da adequação da assistência pré-natal em uma unidade tracional da atenção primária à saúde. Revista de Enfermagem do Centro-Oeste Mineiro, 2014.

POLGLIANE, Rúbia Bastos Soares et al. Adequação do processo de assistência pré-natal segundo critérios do Programa de Humanização do Pré-natal e Nascimento e da Organização Mundial de Saúde. Ciência \& Saúde Coletiva, v. 19, p. 1999-2010, 2014.

REDE Interagencial de Informação para a Saúde (RIPSA). Indicadores e dados básicos para a saúde no Brasil (IDB): Conceitos e critérios. RIPSA (Rede interagencial de informações para a saúde). Disponível em < http://www.ripsa.org.br/vhl/indicadores-edados-basicos-para-a-saude-no-brasil-idb/conceitos-e-criterios/> Acesso em 19 de maio de 2017.

SERRUYA, Suzanne Jacob et al. O panorama da atenção pré-natal no Brasil e o Programa de Humanização do Pré-natal e Nascimento. Revista Brasileira de Saúde Materno Infantil, 2004. 